\title{
Ueber Complication von Schwangerschaft, Geburt und Wochenbett mit Wandermilz.
}

Von

\author{
Frauenarzt Dr. Karl Heil in Darmstadt.
}

Wenn man mit Litten (1) die Wandermilz definirt als eine dauernde Dislocation der Milz, welche mit einem abnormen Grad von Verschieblichkeit verbunden ist, so müssen alle diejenigen Fälle ausgesondert werden, bei denen eine an normaler Stelle gelegene Milz nach den verschiedenen Richtungen hin innerhalb mässiger Grenzen verschoben werden kann oder bei Lageveränderungen eine Verschiebung eingeht (passiv verschiebliche Milz). "Die eigentliche Wandermilz ist dadurch ausgezeichnet, dass sie an normaler Stelle fehlt und an einer ihr nicht zukommenden dauernd angetroffen wird."

Nach dieser Definition sind die Fälle geringerer Verschieblichkeit, bei denen es durch die in Folge der Schwangerschaft physiologisch eintretende Schwellung der Milz und durch die Lockerung der Bandapparate in der Schwangerschaft nach Schäffer (2) leicht zum Tiefertreten der Milz kommen kann, eigentlich nicht als ${ }_{7}$ Wandermilz" anzusprechen.

Fälle von Wandermilz im strengen Sinne, die Schwangerschaft, Geburt und Wochenbett compliciren, scheinen in der That recht selten zu sein, wenigstens ergiebt die Durchsicht der Literatur nur eine geringe Ausbeute.

In der Schwaugerschaft kann eine Wandermilz zunächst zu diagnostischen Irrthümern Veranlassung geben oder sie kann differentialdiagnostische Schwierigkeiten bezüglich einer bestehenden Schwangerschaft machen. Ferner können die durch eine Wandermilz verursachten Beschwerden, die nach Bessel-Hagen (3) vorzugsweise durch Zerrung des Magens und der Bauchspeicheldrüse 
ausgelöst werden, den Verlauf der Schwangerschaft beeinflussen. Diese subjectiven Störungen sind nach ihrer Intensität allerdings ansserordentlich verschieden und kömnen bisweilen so gering sein, dass die Patientin von ihrem Leiden gar nichts weiss. In anderen Fällen werden neben localen Schmerzen auch solche geklagt, die in die Schenkelgegend oder nach dem Magen oder der Herzgegend hin ausstrahlen. Bei der diagnostischen Werthung allgemeiner Beschwerden weist Litten darauf hin, dass solche ganz ähnlicher Art sich auch bei Hysterischen und Frauen mit Genitalleiden wiederfinden. Die diagnostischen Schwierigkeiten werden durch mehrere Beobachtungen illustrirt. So theilt Litten einen Fall von Rezek mit, den auch $\nabla$. Engel (4) citirt, wo es sich um eine Frau handelte, die sich schwanger glaubte. „Der Unterleib hatte in der Mittellinie das Ansehen eines Bauchbruches. Bei der Palpation fühlte man durch die normale Bauchwand eine Geschwulst hart und glatt, nicht elastisch, leicht beweglich, nach oben convex, an dem unteren Rande mehr nach links eine Furche. Das Leiden wurde anfangs für Bauchschwangerschaft, später für Ovarialtumor gehalten. Bei genauer Untersuchung fand Rezek, dass die Geschwulst nach allen Seiten versehiebbar war, sowohl in das rechte, wie linke Hypochondrium gedrängt werden konnte, mit den Organen des kleinen Beckens nicht zusammenhing. Eine Wandermilz wurde ihm um so wahrscheinlicher, als er erfuhr, dass Patientin seit fünf Jahren öfters an Intermittens gelitten, in der Milzgegend darnach eine harte Geschwulst gefühlt hatte, die vor fünf Monaten nach einem Fall von einer Treppe sich in den Bauchraum gesenkt habe."

Bei dem dritten von v. Engel (4) mitgetheilten Fall gab die Wandermilz gleichfalls Veranlassung zu einer Fehldiagnose. Die Frau hatte einmal ohne Störung geboren. Die in das kleine Becken hinabgesunkene Wandermilz hatte bei der ersten Untersuchung einen graviden Uterus rom 6 . Monat vorgetäuscht. Auch die $\mathrm{Pa}-$ tientin selbst hatte sich für schwanger gehalten, da die Periode lange - im Ganzen 16 Monate - ausgeblieben war, und hatte Kindsbewegungen zu spüren geglaubt. Die Wandermilz war nach einem Sturz von einer Leiter manifest geworden.

v. Engel macht darauf aufmerksam, dass die in das kleine Becken gesunkene $\mathrm{Milz}$ den Uterus retrodeviiren kann.

v. Engel citirt auch einen Fall von Dietl, bei dem die Differentialdiagnose quoad graviditatem schwierig war. 
Wenn auch Litten schreibt, dass Schwierigkeiten, eine dislocirte Milz von einer Ovariengeschwulst zu unterscheiden, bei den modernen Untersuchungsmethoden kaum vorkommen werden, so beweist eine Mittheilung, die Schauta (5) in der geburtshülflichgynäkologischen Gesellschaft in Wien gemacht hat, dass selbst dem erfahrensten Diagnostiker in dieser Beziehung ein Irrthum unterlaufen kann. Bei einer im III. Monat Graviden machte ein Tumor, der als Ovarialtumor imponirte, Beschwerden. Bei der Laparotomie erwies sich der Tumor als Wandermilz mit sehr langem Stiel und $272 \mathrm{~g}$ Gewicht. Die Schwangerschaft verlief ohne jede Störung weiter.

Dass die Milzexstirpation in der Schwangerschaft ohne Schädigung des Allgemeinbefindens und ohne nachtheiligen Einfluss auf die Schwangerschaft ansgeführt werden kann, beweisen ausser dieser Schauta'schen Beobachtung die Fälle von BlandSutton (6), der eine grosse Wandermilz exstirpirte ohne Schwangersehaftsunterbrechung, von Savor (7) (Exstirpation wegen traumatischer Milzruptur), von Tridandoni (8) und Ullmann (9).

Bei den letzten beiden handelte es sich um Stieldrehung der Wandermilz, wodurch die Indication zur Operation gegeben wurde. Im Falle Tridandoni's hatte sich die Wandermilz während der Schwangerschaft in Folge der Stieldrehung vergrössert und verursachte peritonitische Erscheinungen. Ullmann beobachtete eine 30 jährige III gravida am Ende des IV. Monats. Tor einem halben Jahre wurde nach einer Pleuritis über Schmerzen links im Unterbauch geklagt; daselbst constatirte man eine Geschwulst. Jetzt fand man bei heftigen Schmerzen den Tumor rechts im Hypogastrium, doppelt so gross als früher der linksseitige Tumor war. Laparotomie im rechten Hypochondrium; Milztumor mit einem um $180^{\circ}$ gedrehten Stiel; Ligirung. Zunächst verlief die Schwangerschaft ungestört, aber 7 Wochen nach der Entlassung verstarb die Patientin an Sepsis in Folge kriminellen Abortes.

Die Stieltorsion der Wandermilz stellt jedenfalls die ernsteste Complication dar und ihre rationelle Behandlung kann nur in der Splenectomie bestehen, worauf wir noch zurückkommen werden.

Andere Beobachtungen, zu denen meine eigenen, später mitzutheilenden, gehören, zeigen aber auch, dass eine Wandermilz, von etwaigen subjectiven Beschwerden abgesehen, den 
Verlauf der Schwangerschaft gar nicht oder kaum be. einflusst.

Hierher gehört der 1. Fall von v. Engel. Es handelte sich um eine 18 jährige I gravida. Während der Schwangerschaft wurde über ziehende, drückende Schmerzen in der linken Seite geklagt. "Der schwangere und somit vergrösserte Uterus drängte die überliegende Wandermilz allmälig in ihre regelmässige Stelle zurück und so konnte die Milz auf die Schwangerschaftsdauer nicht störend einwirken."

Im 2. Falle v. En gel's war die Wandermilz in der vierten Schwangerschaft bemerkt worden, stieg während der Schwangerschaft in die Höhe und verschwand in den letzten Monaten. Nach der Geburt erschien die Geschwulst wieder im Bauch und zwar rechts unten.

In .den drei v. Engel'schen Fällen lag Malariamilz vor.

Auf eine Beobachtung von C. G. Hubbard (10) haben wir beim "Wochenbett" näher einzugehen; auch hier verlief die Schwangerschaft bei einer durch Intermittens bis auf $15-20 \mathrm{~cm}$ im Durchmesser vergrösserten, frei beweglichen Milz bis zum normalen Ende.

Eine Mittheilung von Ahlfeld (11) ist noch za erwähnen, wo es sich um einen pseudoleukämischen Milztumor handelte. „Der schwangere Uterus drängte den Tumor zur Seite, die Frau trug aus und Störungen in der Geburt waren nicht nachzuweisen. Am 6. Tage des Wochenbettes lag der Milztumor der linken Darmbeinschaufel wieder auf."

Der Vollständigkeit halber ist auch der Fall von sarkomatöser Wandermilz anzuführen, bei dem Wagner (12) die Splenectomie mit Erfolg ausführte. Bei einer 27 jährigen Patientin wurde zuerst in der III. Gravidität ein kleiner Knoten im Leibe bemerkt, der rasch wuchs. Bei der Untersuchung fand sich ein kindskopfgrosser, harter, im Abdomen frei beweglicher, nicht vom Genitalsystem ausgehender Tumor mit scharfem Rande und platter, höckeriger Oberfläche. Der bis ins linke Hypochondrium zu verfolgende Stiel wurde per Koeliotomiam unterbunden und der Tumor abgetragen. Der Tumor wog $1285 \mathrm{~g}$ und glich einer vergrösserten Milz, ohne dass sich Milzgewebe nachweisen liess. Die mikroskopische Untersuchung ergab Rundzellensarkom.

Von einer Störung des Geburtsverlaufs durch gleichzeitig vorhandene Wandermilz weiss die spärliche Casuistik nichts zu berichten. In den beiden Fällen von Engel, denjenigen von 
Hubbard, Ahlfeld und meinem eigenen verlief die Geburt spontan. Dass meine Patientin bei ihrer ersten Niederkunft mit der Zange entbunden wurde, hatte mit der Wandermilz sicher nichts $z u$ thun. Störungen der Nachgeburtsperiode sind gleichfalls keine mitgetheilt. Auch Schäffer (2) ist nichts davon bekannt, dass die von ihm beschriebene, durch die Schwangersehaft erworbene Mobilität der Milz zu Geburtsstörungen geführt hätte. Benking (13) schreibt zwar, dass Geburtsstörungen durch die Nachbarorgane: Blase, Darm, Niere, Milz und Leber verursacht werden können, sofern diese letzteren durch pathologische Veränderung ein mechanisches Hemmniss bilden oder selbst in das kleine Becken herabtreten. Dass die Milz bis in's kleine Becken dislocirt sein kann, beweist die von Litten angeführte Beobachtung Martin's, der die normal grosse Milz von der Vagina aus gelegentlich auch ohne combinirte Untersuchung fühlen konnte. Bei derartigen sehr seltenen Fällen wird aber entweder die verlagerte Milz gleichfalls im Laufe der Gravidität von dem wachsenden Uterus nach oben gedrängt werden und dann die Geburt selbst nicht mehr stören oder es wird frühzeitig zu Einklemmungserscheinungen kommen, die vielleicht zu einer Schwangerschaftsunterbrechung führen.

Auch das Wochenbett scheint durch eine Wandermilz als solche nicht weiter ungünstig beeinflusst $\mathrm{zu}$ werden. Mehrmals ist angegeben, dass nach der Entleerung des Uterus der Milztumor wieder herabstieg und im Wochenbett den Beckenschaufeln auflag.

Bei der schon erwähnten Patientin Hubbard's (10) erfolgte die III. Geburt zur rechten Zeit in Folge Wehenschwäche nach 40 stündigem Kreissen spontan. 8 Stunden post partum trat der Exitus ein, nachdem 5 Stunden post partum sich eine Ohnmacht eingestellt hatte. In der Milz fand sich ein $15 \mathrm{~cm}$ langer, $6,6 \mathrm{~cm}$ breiter Riss. Hubbard nimmt an, dass der Riss nicht während des Geburtsactes entstanden sei.

Es bandelte sich um eine Malariamilz und die pathologische Veränderung des Milzgewebes muss wohl in erster Linie ätiologisch für die Ruptur verantwortlich gemacht werden, wenn es ja auch nicht ausgeschlossen ist, dass die Dislocation und freie Beweglichkeit des Milztumors als unterstützender Factor in Frage kommt.

Dass die durch Malaria vergrösserte und histologisch veränderte Milz sehr zur Ruptur disponirt ist, lehren die Beobachtungen von Schwing (14), Saxtorph, Wilson, Sidey und Simpson (15). 
$O b$ es sich bei den 3 Fällen Simpson's gleichzeitig um Wandermilz gehandelt hat, ist aus dem Referat nicht ersichtlich.

Der von mir selbst beobachtete Fall bietet nun in mehrfacher Hinsicht so viel Interessantes, dass er eine ausführliche Wiedergabe verdient.

Frau N. aus D., damals 28 Jahre alt, suchte mich, am 24.2 . 05 zum ersten Mal in meiner Sprechstunde anf und klagte über starke Kreuzschmerzen. Sie hatte vor 4 Jabren einmal abortirt und hatte vor 2 Jahren - 5. 5. 03 - geboren. Sie wurde durch Zange von einem lebenden Knaben entbunden, der mit Syndaktylie an Händen und Füssen behaftet ist und an einem Unterschenkel eine amniotische Schnürfurche anfweist. Pat. litt bereits vor dieser Geburt an einer Wandermilz und hatte sich wegen der durch diese verursachten Beschwerden im Mai 1902 von Geh.-Rath Czerny in Heidelberg die Splenopexie ausführen lassen. Die Splenopexie war ohne dauernden Erfolg geblieben und die Milz war angeblich seit einiger Zeit wieder ,gerutscht:". Die Genitaluntersuchung ergab einen normal grossen, retroflectirten Uterus und gesunde Adnexe. Der untere Rand der stark vergrösserten Milz reichte bis 3 Querfinger breit unter den Nabel. Das ganze Organ war leicht in toto nach oben verschieblich.

Da. Patientin eine erneute operative Behandlung ablehnte, so rieth ich ihr eine Leibbinde mit Pelotte zu tragen.

Die Menstruation verlief ohne besondere Beschwerden.

Am 5. 7. 06 sah ich Patientin wieder. Sie hatte bisher von ihrer Wandermilz keine besonders grossen Beschwerden.

Am 27. 12. 05 war sie zum letzten $\mathrm{Hal}$ regelmässig menstruirt gewesen: im Januar hatte sich nochmals eine geringe Blutung gezeigt.

Die Gravidität - 6. Monat - war bisher ganz beschwerdelos verlaufen.

Der Fundus uteri stand gerade in Nabelhöhle. Unmittelbar über dem Nabel bemerkt man eine deutlich ausgeprägte, querverlaufende Furche, oberhalb welcher der gleichfalls querverlaufende untere Milzrand deutlich palpabel ist. Der linke Milzpol ist in der linken Axillarlinie zu fühlen. Der Milztumor ist in seiner Mitte etwas druckempfindlicb. Der fernere Verlauf der Schwangerschaft war nach gütiger Mittheilung des Herrn Collegen Marx-Hier, dem ich auch die Angaben über den Geburtsverlauf verdanke, ungestört.

Am 12. 10. 06 erfolgte ziemlich rasch die Spontangeburt eines kräftigen, wohl entwickelten Kindes. Nachgeburt und Wochenbett boten keinerlei Störungen.

Am 15. 10. 06, also am 4. Wochenbettstag, sah ich Frau N. zum letzten Mal. Der Milztumor war deutlich dem oberen Rande des puerperalen Uterus aufliegend zu fühlen.

Von besonderem Interesse ist nun, dass die Zwillingsschwester der Patientin gleichfalls mit einer Wandermilz behaftet ist und zweimal - 1902 und am 14. 10. 1905 - geboren hat.

Ueber die am 18.8. 1902 erfolgte erste Entbindung verdanke ich genaue Aufzeichnungen des damals behandelnden Arztes, Herrn 
Dr. Feuchtwanger-Frankfurt a./M., der freundlichen Vermittelung des Herrn Collegen Marx.

Frau D., I para; während der Schwangerschaft häufige Genitalblutungen, auch blutige Secretion aus den Brüsten.

16. 8. 02. Seit beute Morgen Erbrechen und geringe Schmerzen jm Leib; beides lässt gegen Abend nach. Geburt hat noch nicht begonnen, Kind liegt quer.

17. 8. 9 Uhr Vorm. Wasserabfluss, Muttermund für einen Finger durchgängig. Kopf rechts oben, Steiss links auf der Beckenschaufel. Linke Seitenlage und Druck auf das Beckenende durch untergeschobenes Polster. Keine Wehen.

Abends 6 Uhr liegen kleine Theile vor; seit Vorm. geringer Blutabgang. Kein Placentargewebe zu fühlen.

18. 8. Morgens 2 Uhr. Verschlechterung der kindlichen Herztöne. Cervicalcanal beinahe entfaltet, Nluttermund für einen Finger durchgängig; neben kleinen Theilen (Ferse) eine schwach pulsirende Nabelschnurschlinge.

3 Uhr Morgens. Metreuryse mit ca. $300 \mathrm{ccm}$ Flüssigkeit. 1/2 stündig 0,25 Chinin. Wehen besser. Blutung steht. 3 Pfund.

8 Uhr Vorm. Metreuryse mit 2 Pfund Gewichtszug, von 9 Uhr ab

Kurz darauf wird der Metreurynter geboren und gleich hinterher ein Fuss bis zum Knöchel. Nach einer Weile wird der Fuss weiter vorgepresst und daneben fällt die nur noch schwach pulsirende Nabelschnur vor. Rasche Extraction, wobei der Nabelschnurpuls erlischt. Das Neugeborene wird wieder belebt und bleibt am Leben.

Nach der Geburt des Kindes mässige Blutung. Expression der Placenta. 1,5 Extract. Secal. cornut. Die hohe Dosis war irrthümlich gegeben; die Frau klagte am nächsten Tage über starkes Kribbeln in den Beinen. Wegen andauernder Blutung Einstellen der Cervix im Spiegel, wobei sich grössere Einrisse nicht nachweisen lassen. Gazetamponade von Cervix und Vagina, wonach die Blutung zunächst steht - bis 7 Uhr Abends. bis 140 .

Um 9 Uhr Abends nocbmals starke Blutung, kleiner Puls, 130

19. 8. Blutung steht. Gaze entfernt.

Bis 24. 8. rein blutige Lochien.

Vom 28. 8. ab Lochien nicht mebr blutig. Weiterer Verlauf ungestört.

Sogleich post partum constatirte der College einen ca. kindskopfgrossen Milztumor, der sich bis auf die Beckenschaufel gegen die Mitte des Leibes hin senkte. Versuche, die Milz mittelst Binden und Pelotten an normaler Stelle zurückzuhalten, waren erfolglos.

Nachdem sich Pat. vom Wochenbett erholt hatte, befand sie sich dauernd wohl.

Die 2. Geburt, am 14. 10. 05, verlief glatt.

Immerbin bemerkenswerth ist auch noch, dass eine Tante mütterlicherseits vor einer längeren Reihe von Jahren, nachdem sie vorher zwei Geburten durchgemacht hatte, wegen einer traumatischen Wandermilz von Herrn Geh.-Rath Czerny mit bestem 
Erfolg durch Exstirpation der Milz geheilt wurde. Nach der Milzexstirpation hat diese Frau noch einmal geboren. (Angabe der Nichte, Frau N.)

Dass $\mathrm{Zwillingsscbwestern}$ an Wandermilz leiden, beide ohne nachweisbares Trauma, dürfte für die Aetiolgie dieser Anomalie insofern von Belang sein, als die Annahme berechtigt erscheint, dass es sich hier um congenitale Veränderungen des Bandapparates der Milz gehandelt hat. Malaria kommt bei beiden Frauen m. W. nicht in Frage; es handelt sich also um idiopathische Milzvergrösserung, die vielleicht theilweise secundär bedingt ist durch die primäre Dislocation mit durch diese verursachten Circulationsstörungen.

Das Recidiv nach der Splenopexie, die nicht allzulange vor der ersten Schwangerschaft von Meisterhand ausgeführt worden war, ist wahrscheinlich durch die bald eingetretene Gravidität mit verursacht worden. Ob der vor der Splenopexie erfolgte Abortus mit der Wandermilz in Beziehung gebracht werden kann, muss ich dahingestellt sein lassen. Ebenso will ich nicht entscheiden, ob bei der ersten Entbindung der Zwillingsschwester, Frau D., die Querlage der Frucht durch den Milztumor verschuldet worden ist, wenn auch diese Möglichkeit nicht von der Hand zu weisen sein dürfte.

Da die Wandermilz nach Grawitz (16) eine an und für sich seltene Krankheit ist, so kann es nicht Wunder nehmen, dass die Casuistik der Complication von Schwangerschaft, Geburt und Wochenbett mit dieser Anomalie recht spärlich ist.

Von praktischer Wichtigkeit wird diese Complication fast ausschliesslich während der Gravidität und kann vor Allem die Stieldrehung von ernstester Bedeutung werden.

Wie die mitgetheilten Fälle lehren, giebt die Splenectomie sehr gute Erfolge, auch bezüglich der Fortdauer der Schwangerschaft, so dass die operative Indicationsstellung durch eine gleichzeitig bestehende Gravidität als solche nicht beeinflusst wird.

Der Vorschlag von v. Engel (4), eine Malariawandermilz durch manuelle Stieltorsion zur Atrophie zu bringen, kann im Hinblick auf die bekannten, grossen Gefahren, die gerade bei Stieldrehung durch Nekrose und Erweichung des Organs eintreten können, gar nicht mehr ernstlich discutirt werden.

Therapeutisch wird künftig bei Stieltorsion, ebenso wie in den Fällen, bei denen die Wandermilz an sich sehr hochgradige 
subjective Beschwerden verursacht, nur noch die Entfernung der Milz, die Splenectomie, in Frage kommen können, da die Erfolge der Splenopexie, die obendrein einen technisch viel schwierigeren Eingriff darstellt, viel zu unzuverlässig sind.

Bessel-Hagen räth sogar der Gefahr einer Stieldrehung durch die Splenectomie vorzubeugen, sobald eine Wandermilz diagnosticirt ist, da er in der Totalexstirpation der Wandermilz eine ebenso gefahrlose wie einfache und sicher Erfolg versprechende Operation erblickt.

Im Einzelfall wird sich auch bei der mit einer Schwangerschaft gepaarten Wandermilz die Indicationsstellung nach den sonst giltigen und bereits ziemlich scharf umschriebenen Regeln zu richten haben.

Was für die Stieldrehung der Wandermilz in der Schwangerschaft gilt, besteht ebenso zu Recht für diese Complication im Wochenbett; ein derartiger Fall scheint allerdings bis jetzt noch nicht beschrieben worden zu sein.

\section{Literatur.}

1. Litten, Die Krankheiten der Milz. Nothnagel's spec. Pathol. u. Ther. Bd, 8.

2. Schäffer, v. Winckel's Handbuch. II. Bd. 2. Theil.

3. Bessel-Hagen, Beitrag zur Milzchirurgie. Arch. f. klin. Chir. Bd. 62.

4. v. Engel, Centralbl. f. Gyn. 1886.

5. Schauta, Monatsschr. f. Geb. u. Gyn. Bd. XXIV. Heft 2. S. 275.

6. Bland Sutton, Lancet. Februar 1901.

7. Savor, Centralbl. f. Gyn. 1898.

8. Tridandoni, Annali di obstetr. e gin. 1903. Ref. Jahresberichte. XVII. S. 712 .

9. Ullmann, Klin.-therap. Wochenschr. 1904. S. 42.

10. C. G. Hubbard, New York med. journ. 1879. Ref. Centralbl. f. Gyn. 1879. S. 500.

11. Ahlfeld, Lehrbuch der Geburtsh. II. Aufl. S. 263.

12. Wagner, Chirurgencongress 1894. Monatsschr. f. Geb. u. Gyn. Bd. I. Heft 1. S. 73.

13. Benking, Diss. ing. München 1898. Geburtsstörungen durch nicht sexuelle Erkrankungen Kreissender.

14. Schwing, Centralbl. f. Gyn. 1880. No. 13.

15. Simpson, Ref. Schmidt's Jahrbücher. 136. S. 189.

16. Grawitz, Ebstein-Schwalbe's Handbuch. I. S. 913. 